\title{
Sexual healthcare professionals' views on HPV vaccination for men in the UK
}

\author{
Tom Nadarzynski ${ }^{*}$, , Helen E Smith ${ }^{1}$, Daniel Richardson ${ }^{2}$, Elizabeth Ford ${ }^{1}$ and Carrie D Llewellyn ${ }^{1}$ \\ ${ }^{1}$ Brighton and Sussex Medical School, Room 323, Mayfield House, Brighton BN1 9PH, UK and ${ }^{2}$ Brighton and Sussex University \\ NHS Trust, Claude Nicol Centre, Royal Sussex County Hospital, Eastern Road, Brighton BN2 5BE, UK
}

Background: Human Papillomavirus (HPV) vaccination for men could prevent anal cancers amongst men who have sex with men (MSM).

Methods: An e-survey of attitudes towards vaccination for men in the UK was conducted in July-August 2014.

Results: Among 325 sexual health professionals, 14\% were already vaccinating men against HPV, 83\% recommended genderneutral HPV vaccination and 65\% recommended targeting MSM. Over 50\% reported having poor knowledge about the use of HPV vaccine for MSM and the skills to identify MSM likely to benefit from HPV vaccination.

Conclusions: Clear advice and guidelines on HPV vaccine use for men at sexual health clinics are required to ensure equitable opportunities for vaccination.

Although all men are susceptible to Human Papillomavirus (HPV) infection, men who have sex with men (MSM) are at higher risk of HPV-related recurrent genital warts and anal cancers (Machalek et al, 2012). The reasons for this are multifactorial including, higher HPV-infection rates among MSM, lack of protection from female HPV vaccination as well higher incidence of HIV infection in MSM. HIV infection is strongly associated with persistence of HPV and HIV-infected MSM are disproportionately affected with HPVassociated anal cancer (Chow et al, 2014). In the UK, the Joint Committee on Vaccination and Immunisation (JCVI) is currently evaluating both a gender-neutral HPV vaccination, including all schoolaged boys, and targeted HPV vaccination catch-up programmes for high-risk men such as adult MSM to reduce the HPV-related morbidity (Joint Committee on Vaccination and Immunisation (JCVI) [England], 2014). These two strategies are not mutually exclusive and while a gender-neutral strategy would emulate the existing school-age female HPV vaccination programme, the feasibility and acceptability of a targeted HPV vaccination programme for MSM is yet to be established.

Sexual health clinics have been proposed as a potential setting to offer MSM-targeted HPV vaccination because of their existing and related functions, such as screening for sexually transmitted infections and provision of hepatitis vaccination to MSM. Consequently, there is a need to examine the views on HPV vaccination for MSM amongst sexual healthcare professionals (SHCPs) to measure the potential support for an MSM-targeted HPV vaccination programme. Unlike gender-neutral vaccination in schools, a targeted strategy would require high acceptability rates from both MSM and vaccination providers in order to achieve optimal uptake.

In the US, where MSM below the age of 26 years are eligible for HPV vaccination, the strength of doctors' recommendation is a predictor of HPV vaccine uptake. MSM who believed their doctor would recommend HPV vaccination were $\sim 13$ times more likely to accept it (Reiter et al, 2010). Similarly, $80 \%$ of MSM at a sexual health clinic reported doctor's recommendation as a reason for accepting HPV vaccination (Thomas and Goldstone, 2011). Therefore, we wished to explore the recommendations and attitudes towards HPV vaccination for men among SHCPs in the UK to assess potential acceptability and identify barriers towards HPV vaccination for MSM.

\section{MATERIALS AND METHODS}

An e-survey, containing six demographic questions and 18 attitudinal statements about the use and value of HPV vaccination in men, was conducted during July and August 2014. Participants' agreement with each attitudinal statement was measured using a five-item Likert scale ('strongly disagree' to 'strongly agree'). The statements were adapted from previous studies examining 
attitudes of health professionals towards HPV vaccination (Riedesel et al, 2005; Hopkins and Wood, 2013), and were further modified after piloting the survey for usability and functionality.

A search of online resources and the databases of three professional organisations (British Association of Sexual Health and HIV, Society of Sexual Health Advisors, National HIV Nurses Association) was undertaken to identify email addresses of UK-based sexual health workers (i.e., consultants, nurses, health advisors). A total of 344 addresses were identified and used to circulate an invitation to participate in the e-survey. Recipients were also asked to distribute the e-survey link to co-workers in their departments. In addition, the invitation to participate was distributed through the professional newsletters of each organisation. No incentive was offered for completion.

Descriptive statistics were used for demographic and attitudinal data. Multiple ordinal regressions tested associations between demographic and attitudinal variables (missing values did not exceed $2.5 \%$ of all responses). Two-tailed Spearman's rank tests were used to examine the relationship between attitudinal variables and the willingness to recommend gender-neutral or MSMtargeted HPV vaccination strategies.

\section{RESULTS}

A total of 325 SHCPs responded (70\% females, 46\% doctors Supplementary Table A). The modal year of gaining clinical qualification was 1993 (s.d.: 9.7). Although respondents came mainly from the South East of England, the sample was geographically dispersed. About $75 \%$ practised in sexual health clinics where more than $10 \%$ of service users were MSM. Approximately $14 \%$ of respondents reported already vaccinating men against HPV without prior recommendations.
Although $84 \%$ agreed to recommend gender-neutral HPV vaccination programme, regardless of sexual orientation, $65 \%$ were supportive of MSM-targeted HPV vaccination (Table 1). Approximately $17 \%$ expressed a lack of favour towards MSM-targeted HPV vaccination strategy. The recommendation of gender-neutral programme was associated with the attitude that HPV vaccination of heterosexual men is valuable and worthwhile (Supplementary Tables B and C). SHCPs that serve a largely heterosexual population were more at 2.55 odds to recommend gender-neutral HPV vaccination (CI: 1.08-6.04). The recommendation of MSM-targeted HPV vaccination was associated with the attitude that MSM would not benefit from female HPV vaccination $(r=-0.30, P<0.002)$ and that targeted HPV vaccination would encourage MSM to engage with sexual health clinics $(r=0.26$, $P<0.002)$. Most respondents were unsure whether other healthcare professionals would recommend the vaccine to MSM.

When respondents were asked to indicate their views on potential MSM-targeted HPV vaccination programmes, about $75 \%$ agreed that MSM would accept HPV vaccine, that HPV vaccination should be offered at no patient cost, and be available in alternative settings, such as GP practices or pharmacists. Approximately half of the respondents agreed that an HPV vaccination programme needs to be available to all MSM, regardless of their age, and not subject to assessment based on individual susceptibility. About $17 \%$ agreed that HPV vaccination would not be effective in sexually active MSM and $5 \%$ thought that targeted HPV vaccination could lead to unsafe sex. Interestingly, 32\% reported having insufficient knowledge about the HPV vaccination for MSM and 22\% reported a lack of skills to identify MSM potentially eligible for HPV vaccination.

SHCPs who were already vaccinating men against HPV were at 0.34 odds to disagree that MSM are not at risk of HPV-related cancers and that MSM-targeted HPV vaccination is worthwhile (CI: $0.20-0.70)$. These health professionals also perceived

Table 1. The proportion of attitudes towards HPV vaccination in men amongst sexual healthcare professionals

\begin{tabular}{|c|c|c|c|}
\hline Statement & $\begin{array}{l}\text { Strongly agree } \\
\text { and agree }\end{array}$ & Neutral & $\begin{array}{l}\text { Strongly disagree } \\
\text { and disagree }\end{array}$ \\
\hline \multicolumn{4}{|l|}{ Vaccination programme recommendation } \\
\hline $\begin{array}{l}\text { I would recommend HPV vaccination of all men, regardless of their sexual orientation } \\
\text { I would recommend targeting MSM to be vaccinated against HPV } \\
\text { Other healthcare professionals would not recommend HPV vaccination to MSM }\end{array}$ & $\begin{array}{r}271(84 \%) \\
207(65 \%) \\
81(25 \%)\end{array}$ & $\begin{array}{r}26(8 \%) \\
60(18 \%) \\
149(45 \%)\end{array}$ & $\begin{array}{l}25(8 \%) \\
53(17 \%) \\
94(30 \%)\end{array}$ \\
\hline \multicolumn{4}{|l|}{ Perceived population at risk } \\
\hline $\begin{array}{l}\text { Vaccinating females against HPV will reduce HPV-related cancers in heterosexual men } \\
\text { Vaccinating females against HPV will reduce HPV-related cancers in MSM }\end{array}$ & $\begin{array}{r}271(84 \%) \\
47(15 \%)\end{array}$ & $\begin{array}{l}38(11 \%) \\
66(20 \%)\end{array}$ & $\begin{array}{c}15(5 \%) \\
210(65 \%)\end{array}$ \\
\hline \multicolumn{4}{|l|}{ Perceived value } \\
\hline $\begin{array}{l}\text { HPV causes too few cancers among heterosexual men to make it worthwhile to offer vaccination } \\
\text { HPV causes too few cancers among MSM to make it worthwhile to offer vaccination } \\
\text { HPV causes too few cancers among HIV-positive MSM to make it worthwhile to offer vaccination }\end{array}$ & $\begin{aligned} 27 & (8 \%) \\
7 & (3 \%) \\
8 & (3 \%)\end{aligned}$ & $\begin{array}{l}66(21 \%) \\
27(8 \%) \\
22(7 \%)\end{array}$ & $\begin{array}{l}230(71 \%) \\
285(89 \%) \\
290(90 \%)\end{array}$ \\
\hline \multicolumn{4}{|l|}{ Perceived health behaviours } \\
\hline $\begin{array}{l}\text { The majority of MSM would be willing to receive the HPV vaccine } \\
\text { HPV vaccination would encourage MSM to engage with sexual health services } \\
\text { Vaccinating MSM could increase the likelihood of unsafe sex }\end{array}$ & $\begin{array}{l}238(75 \%) \\
190(60 \%) \\
14(5 \%)\end{array}$ & $\begin{array}{l}75(23 \%) \\
97(30 \%) \\
41(13 \%)\end{array}$ & $\begin{array}{c}7(2 \%) \\
30(10 \%) \\
266(82 \%)\end{array}$ \\
\hline \multicolumn{4}{|l|}{ Perceived delivery modes } \\
\hline $\begin{array}{l}\text { The patient should cover the vaccine cost } \\
\text { Targeted HPV vaccination should be based on individual assessment of each MSM attending } \\
\text { sexual health clinic } \\
\text { HPV vaccination should be offered to MSM in alternative settings such as GP practices or } \\
\text { pharmacies }\end{array}$ & $\begin{array}{l}18(6 \%) \\
85(26 \%) \\
239(74 \%)\end{array}$ & $\begin{array}{l}52(16 \%) \\
57(18 \%) \\
49(15 \%)\end{array}$ & $\begin{array}{l}252(78 \%) \\
179(56 \%) \\
34(11 \%)\end{array}$ \\
\hline \multicolumn{4}{|l|}{ Perceived expediency } \\
\hline $\begin{array}{l}\text { All MSM, regardless of their age, should be offered HPV vaccination } \\
\text { It is too late to vaccinate against HPV if MSM are already sexually active }\end{array}$ & $\begin{array}{r}162(51 \%) \\
56(17 \%)\end{array}$ & $\begin{array}{l}80(25 \%) \\
77(24 \%)\end{array}$ & $\begin{array}{r}77(24 \%) \\
189(59 \%)\end{array}$ \\
\hline \multicolumn{4}{|l|}{ Perceived capabilities } \\
\hline $\begin{array}{l}\text { I have the skills to identify MSM that would benefit from the HPV vaccine } \\
\text { I feel sufficiently informed about the HPV vaccination for MSM }\end{array}$ & $\begin{array}{l}155(49 \%) \\
143(44 \%)\end{array}$ & $\begin{array}{l}95(29 \%) \\
76(24 \%)\end{array}$ & $\begin{array}{r}72(22 \%) \\
105(32 \%)\end{array}$ \\
\hline
\end{tabular}


themselves to be more knowledgeable about issues related to HPV vaccination and MSM (OR=8.49, CI: 4.05-15.1). Compared with consultants, nurses were more likely to agree with a MSM-targeted HPV vaccination strategy based on individual assessment $(\mathrm{OR}=3.32$, CI: 1.69-5.65), despite reporting feeling uninformed about the use of HPV vaccine specifically for MSM.

\section{DISCUSSION}

Although the majority of SHCPs support gender-neutral HPV vaccination MSM-targeted HPV vaccination is moderately acceptable. SHCPs perceive a need to protect MSM against HPV-related diseases and some have already been vaccinating high-risk men, despite a lack of policy addressing HPV prevention in MSM. However, the age and the source of funding of vaccine recipients were not defined. Because a fifth of respondents were against an MSM-targeted HPV vaccination strategy, these professionals might be less supportive of offering HPV vaccination to their MSM patients if a catch-up programme was introduced. Moreover, more than half of the respondents perceived a lack of adequate knowledge and skills that would allow them to offer HPV vaccination to MSM. Professionals that have already been vaccinating men against HPV reported having sufficient knowledge about HPV vaccine and positive attitudes towards a targeted HPV vaccination strategy. Similar disparities in healthcare professionals' opinions and concerns about the effectiveness of various vaccination strategies have been reported previously (Hopkins and Wood, 2013; Vadaparampil et al, 2014). Thus, in the absence of clear national guidelines on HPV vaccination for men, such variation in attitudes and recommendations is not unexpected.

If MSM-targeted HPV vaccination is recommended in the UK, the motivation of SHCPs will have an important role in achieving the required uptake for an effective vaccination programme (Rosenthal et al, 2011). The lack of active promotion of the HPV vaccine to eligible patients, as observed in the US, could diminish uptake and undermine the potential benefits of this strategy. Therefore, it is essential that in parallel to a vaccination strategy there is investment in the production of clear advice, guidelines and education to ensure that all SHCPs offer HPV vaccination to MSM service users.

This is the first UK study to measure healthcare professionals' attitudes towards MSM-targeted HPV vaccination, and has highlighted the importance of examining the motivation of health professionals involved in vaccination delivery to monitor any impact of attitudes and recommendation on vaccine acceptability and uptake. The sample size is substantial and geographically diverse, but the study lacks the perspective of other professionals likely to be involved in targeted HPV vaccination, such as GPs or pharmacists. Due to online recruitment methods, we do not have any assessment of the response rate or the representativeness of the sample. There is also a possibility of bias by self-selection. Participants' research knowledge was not examined. SHCPs attitudes need to be monitored over time to ensure they remain favourable and supportive of the current policies. Future research should identify the barriers and enablers of potential MSM-targeted HPV vaccination and assess the levels of HPV vaccine acceptability among MSM in the UK.

In conclusion, specific national guidelines for SHCPs on the use of HPV vaccine for men in sexual health clinics are urgently needed to standardise HPV vaccination practices and ensure equitable distribution of health promoting services, so that all men at high risk of HPV are offered vaccination that reduces the risk of anal cancer.

\section{ACKNOWLEDGEMENTS}

We thank members of the British Association of Sexual Health and HIV, the Society of Sexual Health Advisors, and the National HIV Nurses Association for distributing and completing the online questionnaire. Also, thanks to Nicky Perry from Brighton and Sussex University Hospitals Trust and Alex Pollard from Brighton and Sussex Medical School for advice on the study design. We also thank four anonymous reviewers for their useful comments. This work was funded through a competitive $\mathrm{PhD}$ scholarship from the University of Brighton.

\section{CONFLICT OF INTEREST}

The authors declare no conflict of interest.

\section{AUTHOR CONTRIBUTIONS}

TN, CL, DR and HS designed the study. TN and DR were involved in the recruitment and data collection. TN and EF were involved in data analysis. All authors contributed to the drafting of the manuscript and the approval of the final version.

\section{REFERENCES}

Chow EPF, Read TRH, Wigan R, Donovan B, Chen MY, Bradshaw CS, Fairley CK (2014) Ongoing decline in genital warts among young heterosexuals 7 years after the Australian human papillomavirus (HPV) vaccination programme. Sex Transm Infect; e-pub ahead of print 10 October 2014; doi:10.1136/sextrans-2014-051813.

Hopkins TG, Wood N (2013) Female human papillomavirus (HPV) vaccination: Global uptake and the impact of attitudes. Vaccine 31(13): 1673-1679.

Joint Committee on Vaccination and Immunisation (JCVI) [England] (2014) JCVI interim position statement on HPV vaccination of men who have sex with men (MSM). Available at www.gov.uk/government/publications/ interim-statement-on-hpv-vaccination-of-men-who-have-sex-with-men (November 2014).

Machalek DA, Poynten M, Jin F, Fairley CK, Farnsworth A, Garland SM, Hillman RJ, Petoumenos K, Roberts J, Tabrizi SN, Templeton DJ, Grulich AE (2012) Anal human papillomavirus infection and associated neoplastic lesions in men who have sex with men: a systematic review and meta-analysis. Lancet Oncol 13(5): 487-500.

Reiter PL, Brewer NT, McRee AL, Gilbert P, Smith JS (2010) Acceptability of HPV vaccine among a national sample of gay and bisexual men. Sex Transm Dis 37(3): 197-203.

Riedesel JM, Rosenthal SL, Zimet GD, Bernstein DI, Huang B, Lan D, Kahn JA (2005) Attitudes about Human Papillomavirus Vaccine among Family Physicians. J Pediatr Adolesc Gynecol 18(6): 391-398.

Rosenthal SL, Weiss TW, Zimet GD, Ma L, Good MB, Vichnin MD (2011) Predictors of HPV vaccine uptake among women aged 19-26: importance of a physician's recommendation. Vaccine 29(5): 890-895.

Thomas EA, Goldstone SE (2011) Should I or shouldn't I: decision making, knowledge and behavioral effects of quadrivalent HPV vaccination in men who have sex with men. Vaccine 29(3): 570-576.

Vadaparampil ST, Malo TL, Kahn JA, Salmon DA, Lee J-H, Quinn GP, Roetzheim RG, Bruder KL, Proveaux TM, Zhao X, Halsey NA, Giuliano AR (2014) Physicians' human papillomavirus vaccine recommendations, 2009 and 2011. Am Journal Prev Med 46(1): 80-84.

Supplementary Information accompanies this paper on British Journal of Cancer website (http://www.nature.com/bjc) 